\title{
FLORÍSTICA E ESTRUTURA DO COMPONETE ARBUSTIVO-ARBÓREO DE MATA DE RESTINGA ARENOSA NO PARQUE ESTADUAL DE ITAPEVA, RIO GRANDE DO SUL ${ }^{1}$
}

\author{
Robson Santos², Régis Cardoso Silva ${ }^{3}$, Dilton Pacheco ${ }^{4}$, Rafael Martins ${ }^{2}$ e Vanilde Citadini-Zanette ${ }^{2}$
}

\begin{abstract}
RESUMO - Para ampliar o conhecimento sobre as matas de Restinga arenosa no Rio Grande do Sul, foi realizado estudo florístico e fitossociológico do componente arbustivo-arbóreo de um capão de Restinga do Parque Estadual de Itapeva (PEVA). Neste estudo foi usado o método de parcelas, incluindo todos os indivíduos com DAS (diâmetro a altura do solo) mínimo de $2,5 \mathrm{~cm}$ e totalizando uma área de 0,5 ha. A composição florística resultou em riqueza total de 81 espécies. A densidade total arbustivo-arbórea no capão foi de $4.240 \mathrm{ind} / \mathrm{ha}$. A família com maior riqueza específica foi Myrtaceae e, entre as espécies com maior valor de importância, estão Myrsine umbellata Mart. e Sorocea bonplandii (Baill.) W. C. Burger. Na regeneração natural as 10 primeiras espécies acumularam $54 \%$ da regeneração natural total, com índices que variaram de $13,5 \%$ a $0,2 \%$. A elevada riqueza observada evidenciou a contribuição da "Porta de Torres" como importante corredor migratório das espécies atlânticas na vegetação do Rio Grande do Sul, agregando novas informações sobre a florística e estrutura comunitária da Restinga do Rio Grande do Sul. Os resultados apresentados contribuirão para posteriores estudos de dinâmica no PEVA e para programas de manejo, preservação e recuperação ambiental.
\end{abstract}

Palavras-chave: Restinga, Estrutura fitossociológica e Floresta Atlântica.

\section{FLORISTIC AND STRUCTURE OF THE SHRUB-TREE COMPONENT OF SANDY RESTINGA FOREST IN PARQUE ESTADUAL DE ITAPEVA, RIO GRANDE DO SUL STATE, BRAZIL}

\begin{abstract}
To enlarge the knowledge on sandy Restinga forests in Rio Grande do Sul, it was carried out a shrub-tree component phytosociological and floristic studies of a Restinga forest from Parque Estadual de Itapeva (PEVA). In this study, it was used the the plot method, including all the individuals with DAS (soil height diameter) minimum of $2.5 \mathrm{~cm}$ and totaling an area of $0.5 \mathrm{ha}$. The floristic composition resulted in a total richness of 81 species. The shrub-tree total density in the forest was 4,240 individulas/ha. The family with the highest specific richness was Myrtaceae and, among the species with the highest importance value are Myrsine umbellata Mart. and Sorocea bonplandii (Baill.) W. C. Burger. In the natural regeneration the 10 first species accumulated $54 \%$ of the total natural regeneration, with rates from $13.5 \%$ to $0.2 \%$. The high observed richness showed the contribution of "Porta de Torres" as an important Atlantic species migratory corridor in the vegetation in Rio Grande do Sul, adding new information on Restinga community structure and floristic in Rio Grande do Sul. The results will contribute to further studies on PEVA dynamic and to environmental reclamation, preservation and handling programs.
\end{abstract}

Keywords: Sandy dune vegetation, phytosociological structure and Atlantic Forest.

\footnotetext{
${ }^{1}$ Recebido em 21.12.2010 aceito para publicação em 04.06.2012

${ }^{2}$ Universidade do Extremo Sul Catarinense, Herbário Pe Dr Raulino Reitz, UNESC, Brasil. E-mail: <rsa@ unesc.net>, <rfa@unesc.net> $\mathrm{e}<\mathrm{vcz} @$ unesc.net $>$.

${ }^{3}$ Mestrado em Ciências Ambientais, Universidade do Extremo Sul Catarinense, UNESC, Brasil. E-mail: <registrs@ bol.com.br>.

${ }^{4}$ Secretaria Municipal de Cultura, Esporte e Turismo da Prefeitura Municipal de Nova Veneza, SC. E-mail: <diltontur@hotmail.com>.
} 


\section{INTRODUÇÃO}

De modo geral, as restingas apresentam espécies provenientes de outros ecossistemas, que colonizaram esses ambientes em razão da variedade das condições físicas que ali ocorrem (ARAÚJO; LACERDA, 1987; FREIRE, 1990), porém apresentam variações fisionômicas devido às condições distintas do seu ambiente de origem (ASSUMPÇÃO; NASCIMENTO, 2000; MAGNAGO et al., 2010). As espécies vegetais presentes nessas comunidades detêm características que as permitem colonizar solos pobres, arenosos, encharcados ou com alta salinidade e ambientes com ventos constantes (WAECHTER, 1985).

Muitos são os papéis da vegetação de restinga sobre o ecossistema onde está inserida. Alguns autores associam essa vegetação à estabilização do substrato nesses ambientes, como a proteção da ação de ventos, que é considerada importantíssimo modificador da paisagem (LINDEMAN, 1906; RAMBO, 1954; ASSUMPÇÃO; NASCIMENTO, 2000), além de manter a drenagem natural, contribuindo também para a preservação da fauna endêmica e migratória (FALKENBERG, 1999; ROCHA et al., 2005).

Para o Rio Grande do Sul, duas principais rotas de migração de espécies vegetais tropicais foram pelo lado Leste, servindo de corredor para espécies tropicais atlânticas e pelo Noroeste, constituído de espécies tipicamente estacionais (RAMBO, 1950). As espécies tropicais migraram para o Estado pela "Porta de Torres", a estreita planície entre o Planalto Meridional e o oceano Atlântico, descrita por Rambo (1950) como importante via migratória de espécies tropicais do Sudeste do Brasil.

O Município de Torres constitui importante porta de entrada para a vegetação no Rio Grande do Sul, conforme descrito por Rambo (1950). Nesse corredor se destaca o Parque Estadual de Itapeva (PEVA), que vem sofrendo com a ação humana. Apresenta impacto, principalmente, pela introdução de espécies animais e vegetais exóticas, ocupação humana e pressão agrícola, além do crescimento da cidade de Torres nos limites do parque.

Para melhor compreensão das áreas de restingas são necessárias descrições sobre a vegetação que se pode dar, basicamente, por meio de métodos florísticos e estruturais (KENT; COKER, 1992). Dessa forma, a listagem da flora e a caracterização estrutural contribuem para designar e classificar as vegetações litorâneas, além de diferenciar suas fisionomias (SILVA; BRITEZ, 2005; MAGNAGO et al., 2011), fornecendo mais conhecimento sobre as restingas do Sul do Brasil.

Diante dessas questões, este estudo teve por objetivo conhecer a estrutura e composição florística da restinga arbustivo-arbórea no Parque Estadual de Itapeva, em Torres, Rio Grande do Sul, bem como relacionar a composição florística a diferentes estudos realizados na Floresta Atlântica Sul brasileira.

\section{MATERIAL E MÉTODOS}

\subsection{Localização da área}

O estudo foi realizado no Parque Estadual de Itapeva (PEVA), com área total de 1.000 ha, localizado no Município de Torres, litoral Norte do Rio Grande do Sul (29 $20^{\prime} \mathrm{S}$ e $49^{\circ} 45^{\prime} \mathrm{W}$ (Figuras 1 e 2 ). O clima da região, segundo a classificação de Köppen, é do tipo Cfa, mesotérmico úmido, com inverno pouco intenso, enquanto o verão é quente. A precipitação média anual é de $1.535 \mathrm{~mm}$. A temperatura média anual é de $19,3{ }^{\circ} \mathrm{C}$ (INMET, 2009).

\subsection{Metodologia}

Foram alocadas 100 parcelas de $5 \mathrm{~m}$ x $5 \mathrm{~m}$ e incluídos todos os indivíduos com diâmetro do caule à altura do solo (DAS) mínimo de $2,5 \mathrm{~cm}$, totalizando uma área amostral de 0,25 ha. Esse procedimento foi adotado pelo fato de a área estudada constituir um cordão arenoso, ondulado e com dunas móveis próximas à vegetação estudada, seguindo-se recomendações de Felfili et al. (2011). As coletas de dados e de amostras botânicas foram realizadas durante o período de dezembro de 2008 a maio de 2009, considerando-se apenas indivíduos arbustivo-arbóreos. Foram calculados os descritores fitossociológicos de frequência, densidade, dominância e valor de importância, de acordo com Mueller-Dombois e Ellenberg (1974). O material coletado fértil foi depositado no Herbário Pe. Dr. Raulino Reitz (CRI), da Universidade do Extremo Sul catarinense (UNESC). A atualização taxonômica das espécies, e de seus autores, seguiu as proposições de Forzza et al. (2010).

Para cada espécie arbustivo-arbórea foram fornecidas informações sobre grupo ecológico (pioneira, secundária inicial, secundária tardia e clímax) e 
Fonte: SEMA, 2006 (modificado).

Source: SEMA, 2006 (modified).
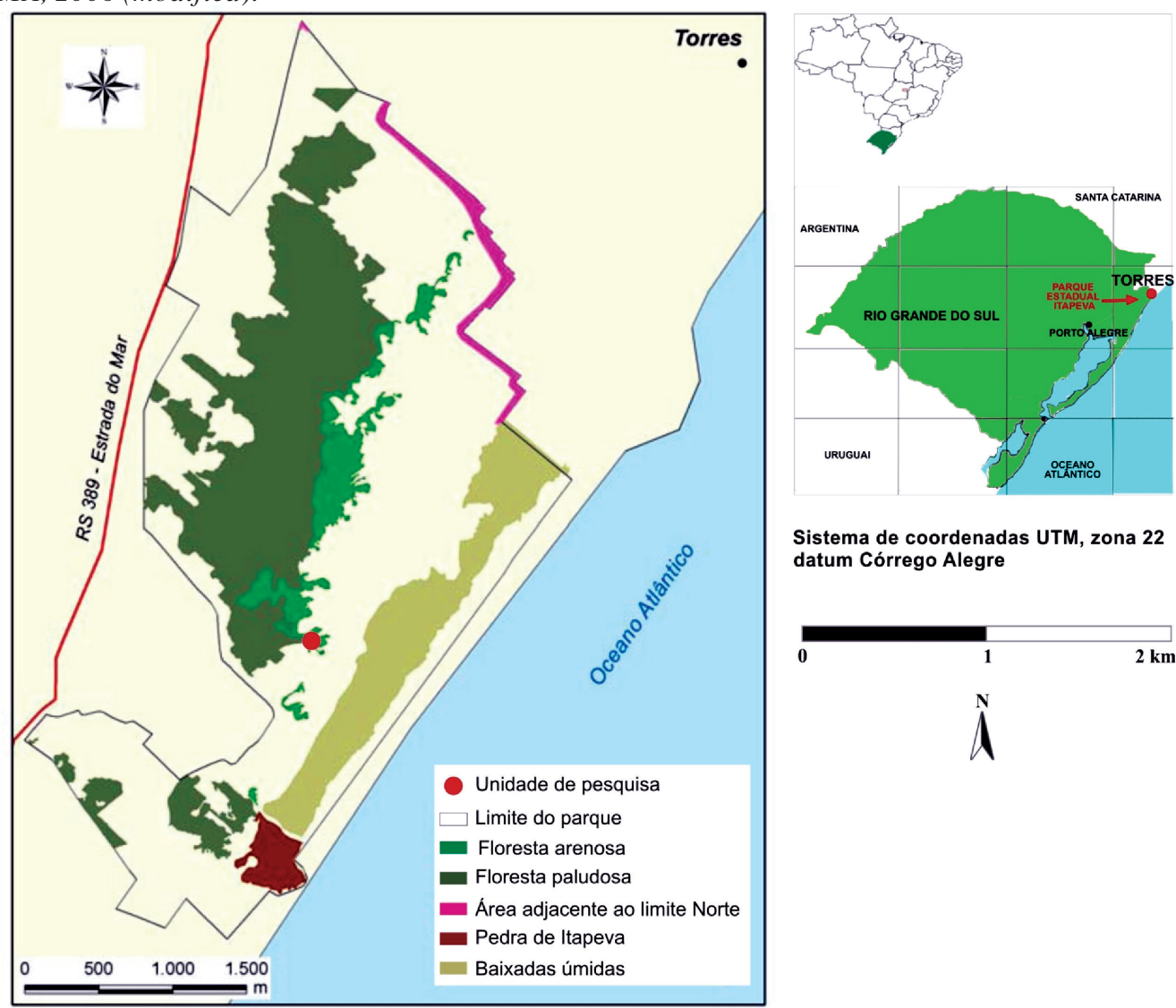

Sistema de coordenadas UTM, zona 22 datum Córrego Alegre

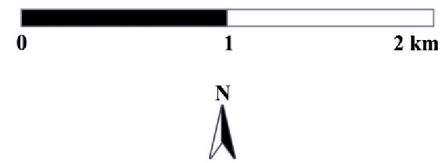

Figura 1 - Localização e classificação das fisionomias presentes no Parque Estadual de Itapeva, município de Torres, Rio Grande do Sul. Fonte: SEMA (2006), modificado.

Figure 1 - Location and physiognomies classification at Parque Estadual de Itapeva, Torres Municipality, Rio Grande do Sul, Brazil.

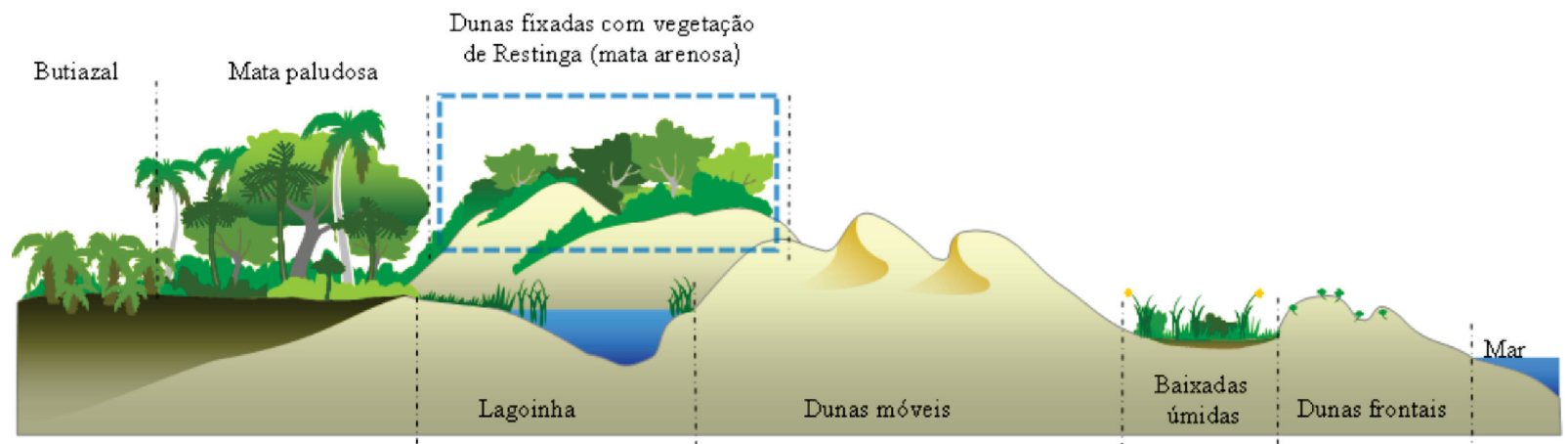

Figura 2 - Perfil esquemático das fitofisionomias presentes no Parque Estadual de Itapeva no município de Torres, RS, em destaque perfil da mata arenosa estudada.

Figure 2 -Design profile of the phytophysiognomies present at Parque Estadual de Itapeva, Torres municipality, RS, Brazil. The studied sandy forest profileis highlighted. 
estratégia de dispersão. Para os grupos ecológicos, seguiu-se a proposta de Ferretti et al. (1995) e para as estratégias de dispersão, os princípios de van der Pijl (1972).

Para estudo da regeneração natural foram estabelecidas, de acordo com Volpato (1994), as seguintes classes: classe 1, composta de indivíduos com altura superior ou igual a $20 \mathrm{~cm}$ e inferior a $50 \mathrm{~cm}$; e classe 2 , formada por indivíduos que apresentaram altura superior ou igual a $50 \mathrm{~cm}$ e DAS inferior a $2,5 \mathrm{~cm}$. A amostragem deu-se em 100 parcelas de $1 \mathrm{~m} \mathrm{x} 1 \mathrm{~m} \mathrm{e}$ $2 \mathrm{~m}$ x $2 \mathrm{~m}$, respectivamente, para cada classe de altura. Foi calculado o potencial de regeneração natural (RN) das duas classes consideradas, que é um parâmetro sintético derivado da abundância (densidade) e da distribuição (frequência) das diferentes classes de tamanho. Também foi estimado o potencial de regeneração natural total (RNT) das espécies, que avalia a regeneração total da espécie através da soma das classes de tamanho consideradas, de acordo com Finol (1971), modificado por Volpato (1994).

Para relacionar a área de estudo e seu padrão de distribuição diante de diferentes estudos realizados em florestas de restinga e áreas de Floresta Ombrófila Densa, foram construídas matrizes de dados binários (0 para ausência e 1 para presença). Para tal finalidade, consultaram-se: Citadini-Zanette (1995) FODS1; CitadiniZanette et al. (2001) REST1; Dillenburg et al. (1992) REST2; Dorneles e Waechter (2004) REST3; Hentschel (2008) REST5; Jarenkow (1994) FODM1; Martins (2005) FODS2; Martins (2010) FODM2; Moraes e Mondin (2001) REST6; Negrelle (2006) FOTB1; este estudo REST4; Rossoni e Baptista (1995) REST7; Scherer et al. (2005) REST8; e Silva (1990) REST9. As matrizes foram analisadas por meio da análise de correspondência (CA), que permite verificar a ocorrência de gradientes na ordenação das amostras (KENT e COKER, 1992), dispostas em um diagrama bidimensional.

\section{RESULTADOS}

Foram registradas 81 espécies, distribuídas em 55 gêneros e 30 famílias botânicas. Foram amostrados 1.060 indivíduos, com densidade total de 4.240 indivíduos.ha-1 (Tabela 1). Das famílias registradas, $13(16 \%)$ estavam representadas por apenas uma espécie, três $(7 \%)$ por duas espécies e oito $(30 \%)$ por três espécies. Myrtaceae apresentou a maior riqueza específica, com
16 espécies (20\%), sendo sete pertencentes ao gênero Myrcia e quatro ao gênero Eugenia. Após, seguiram-se Lauraceae, com 10 espécies (12\%) e Anacardiaceae, Euphorbiaceae e Rubiaceae, com quatro espécies cada (15\%). Essas cinco famílias detiveram $47 \%$ das espécies amostradas.

Foram identificadas 46 espécies arbustivo-arbóreas, que colonizam áreas de floresta arenosa, não citadas no Plano de Manejo do Parque para esse ambiente, além de outras 14 que não haviam sido citadas para o PEVA. São elas: Chrysophyllum viride, Cryptocarya moschata, Diospyros inconstans, Erythroxylum deciduum, Faramea montevidensis, Ficus cestrifolia, Myrceugenia cf. glaucescens, Myrcia campestris, $M$. tijucensis, Nectandra lanceolata, Ouratea salicifolia, Piper gaudichaudianum, Randia ferox e Zanthoxylum astrigerum.

Com relação à densidade, Sorocea bonplandii destacou-se pelo maior número de indivíduos (372 indivíduos.ha-1). Quanto à frequência, Myrsine umbellata apresentou maior ocorrência, estando presente em 44 das 100 unidades amostrais. O valor obtido para área basal total foi de 41,6 $\mathrm{m}^{2} \cdot \mathrm{ha}^{-1}$, sendo a espécie com maior dominância e contribuição nesse parâmetro $M$. umbellata, com 4,64 $\mathrm{m}^{2} \cdot \mathrm{ha}^{-1}$.

Os valores de diâmetro (DAS) variaram de $2,5 \mathrm{~cm}$ até $63,7 \mathrm{~cm}$. Aproximadamente, $44,8 \%$ do total concentraram-se entre 2,5 e 4,9 cm; $53,3 \%$ entre 5,0 e $29,9 \mathrm{~cm}$; e apenas $2 \%$ apresentaram caules iguais ou superiores a $30,0 \mathrm{~cm}$. O maior valor diamétrico foi obtido por Ficus cestrifolia $(63,7 \mathrm{~cm})$, seguido por indivíduos de Chrysophyllum inornatum e Coussapoa microcarpa, com 60,8 cm e 59,5 cm de diâmetro, respectivamente.

As espécies de maior valor de importância (VI) foram Myrsine umbellata, Sorocea bonplandii, Eugenia brevistyla, Chrysophyllum inornatum, Nectandra lanceolata, N. oppositifolia, Myrcia glabra, Aiouea saligna, Coussapoa microcarpa e Cupania vernalis. Essas 10 espécies totalizaram 42,6\% dos indivíduos amostrados.

A altura dos indivíduos registrados neste estudo variou de 1 a $13 \mathrm{~m}$, com a altura média de 4,4 m. As espécies encontradas no estrato inferior da área de estudo foram Sorocea bonplandii, Gymnanthes concolor, Chrysophyllum inornatum, Cupania

Revista Árvore, Viçosa-MG, v.36, n.6, p.1047-1059, 2012 
Tabela 1 - Parâmetros estruturais para as espécies arbustivo-arbóreas em 0,25ha de uma floresta de restinga arenosa no Parque Estadual de Itapeva, Torres, RS, para indivíduos com DAS $\geq 2,5 \mathrm{~cm}$. Onde: H- altura máxima (m), FAfrequência absoluta (\%), DoA- dominância absoluta $\left(\mathrm{m}^{2} \cdot \mathrm{ha}^{-1}\right)$, DA- densidade absoluta (indivíduos.ha-1), VI- valor de importância, Eco- grupo ecológico, onde, P- pioneira; Si- secundária inicial; St- secundária tardia e C- clímax; SD- síndrome de dispersão, onde, An- anemocoria, Au- autocoria e Z- zoocoria.

Table 1 - Structural parameters of shrub-trees species in 0.25ha in a Restinga forest at Parque Estadual de Itapeva, Torres, $R S$, for individuals with DAS $\geq 2,55 \mathrm{~cm}$. Where: $H$ - maximum height ( $\mathrm{m}) ; \mathrm{FA}$-absolute frequency (\%), DoA-absolute dominance $\left(\mathrm{m}^{2} \cdot \mathrm{ha}^{-1}\right)$, DA- absolute density (individuals.ha- $\left.{ }^{1}\right)$, VI- importance value, ECO-ecological group, where, $P$ - pioneer, Si-initial secondary, St-late secondary and C-climax; SD-dispersal syndrome, where, An-anemochoric, Au- autochoric and Z- zoochoric.

\begin{tabular}{|c|c|c|c|c|c|c|c|}
\hline Espécie & $\mathrm{H}$ & FA & DoA & $\mathrm{DA}$ & VI & Eco & $\mathrm{SD}$ \\
\hline Myrsine umbellata Mart. & 13,0 & 44 & 4,639 & 260 & 7,89 & $\mathrm{Si}$ & $\mathrm{Z}$ \\
\hline Sorocea bonplandii (Baill.) W.C.Burger et al. & 6,0 & 41 & 1,162 & 372 & 5,84 & St & $\mathrm{Z}$ \\
\hline Eugenia brevistyla D.Legrand & 9,0 & 33 & 2,967 & 208 & 5,61 & $\mathrm{P}$ & $\mathrm{Z}$ \\
\hline Chrysophyllum inornatum Mart. & 11,0 & 30 & 2,188 & 248 & 5,15 & $\mathrm{C}$ & $\mathrm{Z}$ \\
\hline Nectandra lanceolata Nees et Mart. & 11,0 & 19 & 2,739 & 116 & 4,02 & $\mathrm{C}$ & $\mathrm{Z}$ \\
\hline Nectandra oppositifolia Nees et Mart. & 12,0 & 16 & 2,924 & 84 & 3,78 & St & $\mathrm{Z}$ \\
\hline Myrcia glabra (O. Berg) D. Legrand & 9,0 & 24 & 1,174 & 168 & 3,42 & St & $\bar{Z}$ \\
\hline Aiouea saligna Meisn. & 9,0 & 26 & 1,480 & 124 & 3,42 & $\mathrm{Si}$ & $\mathrm{Z}$ \\
\hline Coussapoa microcarpa (Schott) Rizzini & 11,0 & 11 & 2,706 & 64 & 3,20 & St & $\mathrm{Z}$ \\
\hline Cupania vernalis Cambess. & 7,5 & 27 & 0,756 & 164 & 3,20 & St & $\mathrm{Z}$ \\
\hline Alchornea triplinervia (Spreng.) Müll. Arg. & 12,0 & 15 & 2,037 & 80 & 2,99 & $\mathrm{Si}$ & $\mathrm{Z}$ \\
\hline Ficus cestrifolia Schott & 7,5 & 11 & 2,582 & 44 & 2,95 & $\mathrm{P}$ & $\mathrm{Z}$ \\
\hline Myrcia pubipetala Miq. & 12,0 & 17 & 1,654 & 100 & 2,93 & St & $\mathrm{Z}$ \\
\hline Gymnanthes concolor Spreng. & 6,0 & 17 & 0,259 & 164 & 2,32 & St & $\mathrm{Au}$ \\
\hline Solanum pseudoquina A. St.-Hil. & 12,0 & 14 & 1,187 & 80 & 2,26 & $\mathrm{P}$ & $\mathrm{Z}$ \\
\hline Marlierea eugeniopsoides (Kausel \& D. Legrand) D. Legrand & 10,0 & 12 & 0,965 & 104 & 2,17 & $\mathrm{C}$ & $\mathrm{Z}$ \\
\hline Psidium cattleianum Sabine & 8,0 & 19 & 0,642 & 88 & 2,12 & St & $\mathrm{Z}$ \\
\hline Eugenia bacopari C. D. Legrand & 6,5 & 15 & 0,345 & 128 & 2,01 & $\mathrm{P}$ & $\mathrm{Z}$ \\
\hline Ocotea pulchella (Nees) Mez & 11,0 & 14 & 0,833 & 68 & 1,88 & $\mathrm{C}$ & $\mathrm{Z}$ \\
\hline Matayba guianensis Aubl. & 10,0 & 13 & 0,611 & 76 & 1,72 & St & $\mathrm{Z}$ \\
\hline Maytenus sp. & 13,0 & 14 & 0,194 & 104 & 1,65 & St & $\mathrm{Z}$ \\
\hline Mollinedia schottiana (Spreng.) Perkins & 5,0 & 13 & 0,227 & 100 & 1,60 & $\mathrm{C}$ & $\mathrm{Z}$ \\
\hline Myrcia brasiliensis Kiaersk. & 7,0 & 13 & 0,366 & 60 & 1,39 & St & $\mathrm{Z}$ \\
\hline Zanthoxylum rhoifolium Lam. & 9,0 & 11 & 0,575 & 44 & 1,34 & $\mathrm{Si}$ & $\mathrm{Au}$ \\
\hline Guapira opposita (Vell.) Reitz & 12,0 & 11 & 0,381 & 60 & 1,31 & $\mathrm{Si}$ & $\mathrm{Z}$ \\
\hline Cabralea canjerana (Vell.) Mart. & 7,5 & 12 & 0,257 & 60 & 1,26 & St & $\mathrm{Z}$ \\
\hline Esenbeckia grandiflora Mart. & 5,0 & 10 & 0,070 & 88 & 1,23 & St & $\mathrm{Au}$ \\
\hline Allophylus edulis (A. St.-Hil., Cambess. et A. Juss.) Radlk. & 7,0 & 11 & 0,292 & 48 & 1,14 & $\mathrm{Si}$ & $\mathrm{Z}$ \\
\hline Mollinedia sp. & 4,0 & 8 & 0,096 & 60 & 0,94 & $\mathrm{C}$ & $\mathrm{Z}$ \\
\hline Endlicheria paniculata (Spreng.) J. F. Macbr. & 9,0 & 6 & 0,246 & 52 & 0,90 & St & $\mathrm{Z}$ \\
\hline Symphyopappus casarettoi Robinson & 6,0 & 8 & 0,217 & 40 & 0,87 & $\mathrm{P}$ & An \\
\hline Schinus polygamus (Cav.) Cabrera & 8,0 & 4 & 0,575 & 28 & 0,87 & $\mathrm{P}$ & $\mathrm{Z}$ \\
\hline Schinus terebinthifolius Raddi & 4,0 & 7 & 0,245 & 40 & 0,85 & $P$ & $\mathrm{Z}$ \\
\hline Sapium glandulosum (L.) Morong & 6,0 & 8 & 0,102 & 48 & 0,85 & $\mathrm{P}$ & $\mathrm{Z}$ \\
\hline Myrsine lorentziana (Mez) Arechav. & 7,0 & 8 & 0,169 & 40 & 0,84 & $\mathrm{Si}$ & $\mathrm{Z}$ \\
\hline Ficus luschnathiana (Miq.) Miq. & 11,0 & 3 & 0,623 & 12 & 0,74 & St & $\mathrm{Z}$ \\
\hline Blepharocalyx salicifolius (Kunth) O. Berg & 6,0 & 7 & 0,123 & 36 & 0,72 & $\mathrm{P}$ & $\mathrm{Z}$ \\
\hline Erythroxylum cuspidifolium Mart. & 5,5 & 7 & 0,063 & 40 & 0,70 & $\mathrm{C}$ & $\mathrm{Z}$ \\
\hline Posoqueria latifolia (Rudge) Roem. et Schut. & 4,0 & 8 & 0,051 & 32 & 0,68 & $\mathrm{Si}$ & $\mathrm{Z}$ \\
\hline Garcinia gardneriana (Planch. et Triana) Zappi & 6,0 & 6 & 0,072 & 40 & 0,66 & St & $\mathrm{Z}$ \\
\hline Cryptocarya moschata Nees et Mart. ex Nees & 10,0 & 3 & 0,424 & 16 & 0,61 & $\mathrm{C}$ & $\mathrm{Z}$ \\
\hline Erythroxylum deciduum A. St. Hil. & 7,0 & 5 & 0,261 & 20 & 0,61 & $\mathrm{P}$ & $\mathrm{Z}$ \\
\hline Ouratea parviflora (DC.) Baill. & 4,5 & 7 & 0,029 & 28 & 0,58 & $\mathrm{Si}$ & $\mathrm{Z}$ \\
\hline
\end{tabular}


Tabela 1 - Cont.

Table 1 - Cont.

\begin{tabular}{|c|c|c|c|c|c|c|c|}
\hline Espécie & $\mathrm{H}$ & FA & DoA & DA & VI & Eco & $\mathrm{SD}$ \\
\hline Myrsine coriacea (Sw.) R. Br. ex Roem et Schult. & 6,0 & 5 & 0,202 & 20 & 0,56 & $\mathrm{P}$ & $\mathrm{Z}$ \\
\hline Eugenia schuechiana O. Berg & 5,0 & 6 & 0,091 & 24 & 0,55 & $\mathrm{C}$ & $\mathrm{Z}$ \\
\hline Faramea montevidensis (Cham. et Schltdl.) DC. & 6,0 & 4 & 0,082 & 32 & 0,51 & $\mathrm{C}$ & $\mathrm{Z}$ \\
\hline Casearia sylvestris $\mathrm{Sw}$. & 6,0 & 5 & 0,089 & 24 & 0,50 & $\mathrm{Si}$ & $\mathrm{Z}$ \\
\hline Persea willdenovii Kosterm. & 10,0 & 4 & 0,167 & 20 & 0,48 & St & $\mathrm{Z}$ \\
\hline Randia ferox (Cham. et Schltdl.) DC. & 5,0 & 5 & 0,037 & 24 & 0,46 & $\mathrm{Si}$ & $\mathrm{Z}$ \\
\hline Lithraea brasiliensis Marchand & 4,0 & 4 & 0,078 & 24 & 0,44 & $\mathrm{P}$ & $\mathrm{Z}$ \\
\hline Hirtella hebeclada Moric. ex DC. & 4,5 & 3 & 0,114 & 20 & 0,39 & St & $\mathrm{Z}$ \\
\hline Piper gaudichaudianum Kunth & 4,5 & 4 & 0,036 & 20 & 0,38 & $\mathrm{Si}$ & $\mathrm{Z}$ \\
\hline Prunus myrtifolia (L.) Urb. & 5,0 & 4 & 0,070 & 16 & 0,38 & $\mathrm{Si}$ & $\mathrm{Z}$ \\
\hline Trichilia lepidota Mart. & 8,0 & 3 & 0,076 & 12 & 0,30 & $\mathrm{C}$ & $\mathrm{Z}$ \\
\hline Ocotea puberula (Reich.) Nees & 8,0 & 1 & 0,258 & 4 & 0,29 & $\mathrm{C}$ & $\mathrm{Z}$ \\
\hline Miconia sellowiana Naudin & 7,0 & 3 & 0,050 & 12 & 0,28 & $\mathrm{Si}$ & $\mathrm{Z}$ \\
\hline Persea venosa Nees & 5,5 & 2 & 0,111 & 12 & 0,28 & St & $\mathrm{Z}$ \\
\hline Ilex theezans Mart. ex Reissek & 6,5 & 2 & 0,060 & 12 & 0,24 & $\mathrm{P}$ & $\mathrm{Z}$ \\
\hline Myrceugenia cf. glaucescens (Cambess.) D. Legrand \& Kausel & 5,0 & 1 & 0,199 & 4 & 0,24 & St & $\mathrm{Z}$ \\
\hline Myrcia multiflora (Lam.) DC. & 6,0 & 2 & 0,050 & 12 & 0,23 & $\mathrm{C}$ & $\mathrm{Z}$ \\
\hline Casearia decandra Jacq. & 3,5 & 1 & 0,055 & 16 & 0,22 & $\mathrm{P}$ & $\mathrm{Z}$ \\
\hline Myrcia palustris DC. & 2,0 & 2 & 0,022 & 8 & 0,18 & $\mathrm{Si}$ & $\mathrm{Z}$ \\
\hline Tetrorchidium rubrivenium Poepp. & 3,0 & 2 & 0,017 & 8 & 0,17 & $\mathrm{Si}$ & $\mathrm{Z}$ \\
\hline Myrcia campestris DC. & 5,0 & 2 & 0,011 & 8 & 0,17 & St & $\mathrm{Z}$ \\
\hline Chrysophyllum viride Mart. et Eichler & 3,0 & 1 & 0,017 & 8 & 0,13 & $\mathrm{C}$ & $\mathrm{Z}$ \\
\hline Schinus sp. & 3,0 & 1 & 0,033 & 4 & 0,11 & $\mathrm{P}$ & $\mathrm{Z}$ \\
\hline Erythroxylum argentinum $\mathrm{O}$. E. Schulz & 1,5 & 1 & 0,029 & 4 & 0,10 & $\mathrm{Si}$ & $\mathrm{Z}$ \\
\hline Ilex dumosa Reissek & 7,5 & 1 & 0,031 & 4 & 0,10 & $\mathrm{P}$ & $\mathrm{Z}$ \\
\hline Ocotea silvestris Vattimo & 5,0 & 1 & 0,029 & 4 & 0,10 & $\mathrm{Si}$ & $\mathrm{Z}$ \\
\hline Zanthoxylum astrigerum (R. S. Cowan) P. G. Waterman & 5,0 & 1 & 0,027 & 4 & 0,10 & $\mathrm{Si}$ & $\mathrm{Au}$ \\
\hline Diospyros inconstans Jacq. & 6,0 & 1 & 0,015 & 4 & 0,09 & St & $\mathrm{Z}$ \\
\hline Eugenia uniflora L. & 4,0 & 1 & 0,018 & 4 & 0,09 & $\mathrm{C}$ & $\mathrm{Z}$ \\
\hline Banara parviflora (A. Gray) Benth. & 2,5 & 1 & 0,005 & 4 & 0,08 & $\mathrm{Si}$ & $\mathrm{Z}$ \\
\hline Cedrela fissilis Vell. & 2,5 & 1 & 0,006 & 4 & 0,08 & St & $\mathrm{Au}$ \\
\hline Ilex pseudobuxus Reissek & 4,0 & 1 & 0,003 & 4 & 0,08 & $\mathrm{P}$ & $\mathrm{Z}$ \\
\hline Machaerium stipitatum (DC.) Vog. & 3,5 & 1 & 0,005 & 4 & 0,08 & $\mathrm{P}$ & An \\
\hline Myrcia tijucensis Kiaersk. & 3,0 & 1 & 0,006 & 4 & 0,08 & $\mathrm{C}$ & $\mathrm{Z}$ \\
\hline Psychotria brachyceras Müll. Arg. & 2,0 & 1 & 0,003 & 4 & 0,08 & St & $\mathrm{Z}$ \\
\hline Vitex megapotamica (Spreng.) Moldenke & 2,0 & 1 & 0,004 & 4 & 0,08 & $\mathrm{Si}$ & $\mathrm{Z}$ \\
\hline Campomanesia xanthocarpa $\mathrm{O}$. Berg & 2,5 & 1 & 0,002 & 4 & 0,08 & $\mathrm{P}$ & $\mathrm{Z}$ \\
\hline Ouratea salicifolia (A. St. Hill. et Tul.) Engl. & 3,5 & 1 & 0,002 & 4 & 0,08 & St & $\mathrm{Z}$ \\
\hline Total & & 690 & 41,612 & 4.240 & 100 & & \\
\hline
\end{tabular}

vernalis e Eugenia bacopari. Aproximadamente, 55\% dos indivíduos amostrados ficaram concentrados no estrato inferior. No estrato médio, a maior representatividade deu-se pelos indivíduos de Eugenia brevistyla, Myrsine umbellata, Soroce a bonplandi, Myrcia glabra e Chrysophyllum inornatum. Entre as espécies que se concentraram no estrato superior, destacaram-se Maytenus sp., Myrcia pubipetala e Ficus luschnathiana, com 13, 12 e $11 \mathrm{~m}$, respectivamente.
Analisando a comunidade arbustivo-arbórea em relação aos grupos ecológicos, das 81 espécies encontradas obtiveram-se $23 \%$ de pioneiras, $26 \%$ de secundárias iniciais, $31 \%$ de secundárias tardias e $20 \%$ de clímax. Com relação às estratégias de dispersão, das 81 espécies amostradas, 74 (91\%) apresentaram dispersão zoocórica (Tabela 1).

Na regeneração natural das espécies arbustivoarbóreas (Tabela 2) foram encontradas 67 espécies de 30 famílias botânicas, considerando-se as duas classes

Revista Árvore, Viçosa-MG, v.36, n.6, p.1047-1059, 2012 
de tamanho. Dessas, $31 \%$ foram registradas na classe 1 (plântulas) e $69 \%$ na classe 2 (juvenis). As famílias mais representativas, juntando-se as duas classes de tamanho, foram Myrtaceae com 13 espécies, totalizando 16\% de RNT; e Lauraceae com sete. Algumas espécies só ocorreram no componente de regeneração, como Daphnopsis racemosa, Euterpe edulis, Guarea macrophylla, Luehea divaricata, Myrcia anacardiifolia, Psychotria sp., P. suterella e Zollernia ilicifolia. Sorocea bonplandii deteve o maior valor de regeneração natural (13,5\%), seguida por Esenbeckia grandiflora (7,8\%), Nectandra oppositifolia $(7,7 \%)$, Sapium glandulosum $(6,7 \%)$ e Eugenia brevistyla $(3,4 \%)$. O menor valor $(0,2 \%)$ foi estimado para 15 espécies, presentes somente na classe de altura 2 (juvenis).

A análise de correspondência, aplicada à matriz contendo os dados de estudos realizados no Sul do Brasil, indicou a separação das áreas nos dois primeiros eixos de ordenação (Figura 3). Embora seja considerada técnica descritiva e exploratória, a Análise de Correspondência simplifica dados complexos e produz análises de informações que suportam as conclusões. A separação das áreas indica que o local deste estudo aparece como zona de contato entre as formações de restinga do Rio Grande do Sul e áreas de Floresta Ombrófila Densa do Rio Grande do Sul e Santa Catarina.

Com relação à distribuição geográfica e aos corredores migratórios, obteve-se a maior parte das espécies como de ampla distribuição, com destaque para S. bonplandii e G. concolor, que contribuíram significativamente com a densidade dos indivíduos na área de estudo. Com relação às espécies do contingente atlântico, destacaram-se: Coussapoa microcarpa, Erythroylum argentinum, Esenbeckia grandiflora, Eugenia schuechiana, Faramea marginata, Ficus organensis, Garcinia gardneriana, Guapira opposita, Myrcia glabra, Myrcia multiflora e Nectandra oppositifolia.

\section{DISCUSSÃO}

De acordo com dados disponíveis no Plano de Manejo do PEVA, até o momento são conhecidas 806 espécies de plantas vasculares, com ocorrência confirmada na unidade de conservação. Embora esteja entre as unidades de conservação com a vegetação mais conhecida no Rio Grande do Sul, a riqueza de sua flora certamente ainda está subestimada (SEMA, 2006),
Tabela 2 - Regeneração natural das espécies arbustivo-arbóreas da restinga no Parque Estadual de Itapeva, Torres, Rio Grande do Sul.

Table 2 - The shrub-trees species natural regeneration in a Restinga at Parque Estadual de Itapeva, Torres Municipality, Rio Grande do Sul, Brazil.

\begin{tabular}{|c|c|c|c|}
\hline Espécie & $\mathrm{RNC}_{1}$ & $\mathrm{RNC}_{2}$ & RNT \\
\hline Sorocea bonplandii & 4,82 & 8,72 & 13,54 \\
\hline Esenbeckia grandiflora & 1,54 & 6,25 & 7,79 \\
\hline Nectandra oppositifolia & 4,01 & 3,68 & 7,69 \\
\hline Sapium glandulosum & 4,36 & 2,34 & 6,70 \\
\hline Eugenia neoaustralis & 0,00 & 3,41 & 3,41 \\
\hline Marlierea eugeniopsoides & 1,34 & 1,94 & 3,28 \\
\hline Cupania vernalis & 0,60 & 2,67 & 3,27 \\
\hline Mollinedia schottiana & 2,89 & 0,00 & 2,89 \\
\hline Myrcia pubipetala & 0,47 & 2,41 & 2,87 \\
\hline Psychotria brachyceras & 0,27 & 2,35 & 2,61 \\
\hline Eugenia schuechiana & 0,20 & 2,34 & 2,54 \\
\hline Myrsine umbellata & 1,54 & 0,80 & 2,34 \\
\hline Persea willdenovii & 0,00 & 2,21 & 2,21 \\
\hline Maytenus sp. & 0,00 & 2,00 & 2,00 \\
\hline Aiouea saligna & 0,60 & 1,27 & 1,87 \\
\hline Guapira opposita & 0,20 & 1,47 & 1,67 \\
\hline Alchornea triplinervia & 0,40 & 1,21 & 1,61 \\
\hline Posoqueria latifolia & 0,60 & 1,00 & 1,60 \\
\hline Gymnanthes concolor & 0,40 & 1,14 & 1,54 \\
\hline Psidium cattleianum & 0,47 & 1,07 & 1,54 \\
\hline Daphnopsis racemosa & 0,00 & 1,47 & 1,47 \\
\hline Endlicheria paniculata & 0,60 & 0,80 & 1,40 \\
\hline Trichilia lepidota & 0,60 & 0,74 & 1,34 \\
\hline Allophylus edulis & 0,20 & 1,14 & 1,34 \\
\hline Prunus myrtifolia & 0,00 & 1,27 & 1,27 \\
\hline Solanum pseudoquina & 0,00 & 1,27 & 1,27 \\
\hline Nectandra lanceolata & 0,20 & 0,94 & 1,14 \\
\hline Faramea montevidensis & 0,40 & 0,74 & 1,14 \\
\hline Chrysophyllum inornatum & 0,20 & 0,87 & 1,07 \\
\hline Myrsine coriacea & 0,00 & 1,07 & 1,07 \\
\hline Ocotea pulchella & 0,40 & 0,47 & 0,87 \\
\hline Lithraea brasiliensis & 0,00 & 0,80 & 0,80 \\
\hline Myrcia brasiliensis & 0,40 & 0,40 & 0,80 \\
\hline Persea venosa & 0,40 & 0,40 & 0,80 \\
\hline Hirtella hebeclada & 0,20 & 0,40 & 0,60 \\
\hline Machaerium stipitatum & 0,00 & 0,60 & 0,60 \\
\hline Miconia sellowiana & 0,20 & 0,40 & 0,60 \\
\hline Schinus terebinthifolius & 0,20 & 0,40 & 0,60 \\
\hline Zanthoxylum rhoifolium & 0,00 & 0,60 & 0,60 \\
\hline Guarea macrophylla & 0,54 & 0,00 & 0,54 \\
\hline Symphyopappus casarettoi & 0,00 & 0,54 & 0,54 \\
\hline Erythroxylum cuspidifolium & 0,00 & 0,47 & 0,47 \\
\hline Erythroxylum deciduum & 0,00 & 0,47 & 0,47 \\
\hline Matayba guianensis & 0,00 & 0,40 & 0,40 \\
\hline Myrcia anacardiifolia & 0,20 & 0,20 & 0,40 \\
\hline Piper gaudichaudianum & 0,20 & 0,20 & 0,40 \\
\hline Ilex theezans & 0,00 & 0,34 & 0,34 \\
\hline Euterpe edulis & 0,00 & 0,27 & 0,27 \\
\hline
\end{tabular}

Revista Árvore, Viçosa-MG, v.36, n.6, p.1047-1059, 2012 
Tabela 2 - Cont.

Table-Cont.

\begin{tabular}{lcccc}
\hline Espécie $----\overline{\mathrm{RNC}_{1}}$ & $\overline{\mathrm{RNC}_{2}}$ & $\overline{\mathrm{RNT}}$ \\
\hline Luehea divaricata & 0,00 & 0,27 & 0,27 \\
Myrcia multiflora & 0,27 & 0,00 & 0,27 \\
Psychotria suterella & 0,27 & 0,00 & 0,27 \\
Blepharocalyx salicifolius & 0,00 & 0,20 & 0,20 \\
Cabralea canjerana & 0,00 & 0,20 & 0,20 \\
Casearia decandra & 0,00 & 0,20 & 0,20 \\
Eugenia bacopari & 0,00 & 0,20 & 0,20 \\
Eugenia uniflora & 0,00 & 0,20 & 0,20 \\
Garcinia gardneriana & 0,00 & 0,20 & 0,20 \\
Ilex pseudobuxus & 0,00 & 0,20 & 0,20 \\
Mollinedia sp. & 0,00 & 0,20 & 0,20 \\
Myrcia glabra & 0,00 & 0,20 & 0,20 \\
Myrsine lorentziana & 0,00 & 0,20 & 0,20 \\
Ouratea parviflora & 0,00 & 0,20 & 0,20 \\
Psychotria sp. & 0,00 & 0,20 & 0,20 \\
Tetrorchidium rubrivenium & 0,00 & 0,20 & 0,20 \\
Vitex megapotamica & 0,00 & 0,20 & 0,20 \\
Zollernia ilicifolia & 0,00 & 0,20 & 0,20 \\
\hline Total & 30,85 & 69,15 & 100 \\
\hline
\end{tabular}

afirmação corroborada neste estudo. No PEVA, pelo menos 67 espécies de plantas vasculares estão ameaçadas de extinção e, ou, protegidas por lei no Rio Grande do Sul (RIO GRANDE DO SUL, 2003). Dessas, três espécies foram encontradas no remanescente estudado (Euterpe edulis, Trichilia lepidota e Ocotea silvestris).

O número de espécies encontradas no PEVAencontrase acima do padrão de riqueza esperado para matas de restinga arenosa no Rio Grande do Sul (WAECHTER, 1985; DILLENBURG et al., 1992; ROSSONI; BAPTISTA, 1995; MORAES; MONDIN, 2001; MORETTO; MONDIN, 2002; DORNELES; WAECHTER, 2004; SCHERER et al., 2007). A representatividade de Myrtaceae, assim como dos gêneros Myrcia e Eugenia, segue os padrões de riqueza para o bioma Mata Atlântica, sugerindo afinidade da área de estudo com formações desse bioma (SACRAMENTO et al., 2007; MURRAY-SMITH et al., 2008).

Notou-se afinidade florística da área de estudo (Restinga) com trabalhos realizados em Floresta Ombrófila Densa no Sul do Brasil, principalmente no Sul de Santa Catarina (Figura 3). Observou-se também ausência, neste estudo, de espécies de Sebastiania descritas entre as mais importantes em estudos nas restingas do Rio Grande do Sul, onde, em muitos casos, predomina no componente arbustivo-arbóreo (DILLENBURG et al., 1992; ROSSONI; BAPTISTA, 1995; MORAES; MONDIN, 2001; DORNELES; WAECHTER, 2004; SCHERER et al., 2005). Diferentemente dos estudos citados, neste trabalho atribuíram-se a $S$. bonplandii, M. umbellata e C. inornatum os maiores valores de densidade. $M$. umbellata também não foi citada entre as mais abundantes nas restingas do Estado. Sorocea bonplandii destacou-se como uma das mais importantes espécies em estudos conduzidos em florestas estacionais (JARENKOW; WAECHTER, 2001; GIEHL; JARENKOW, 2008) e ombrófila densa (CITADINI-ZANETTE et al., 2003).

Segundo estudos desenvolvidos por Rambo (1950), a maior parte das espécies tropicais originárias do Norte e Centro do Brasil teriam migrado para o Rio Grande

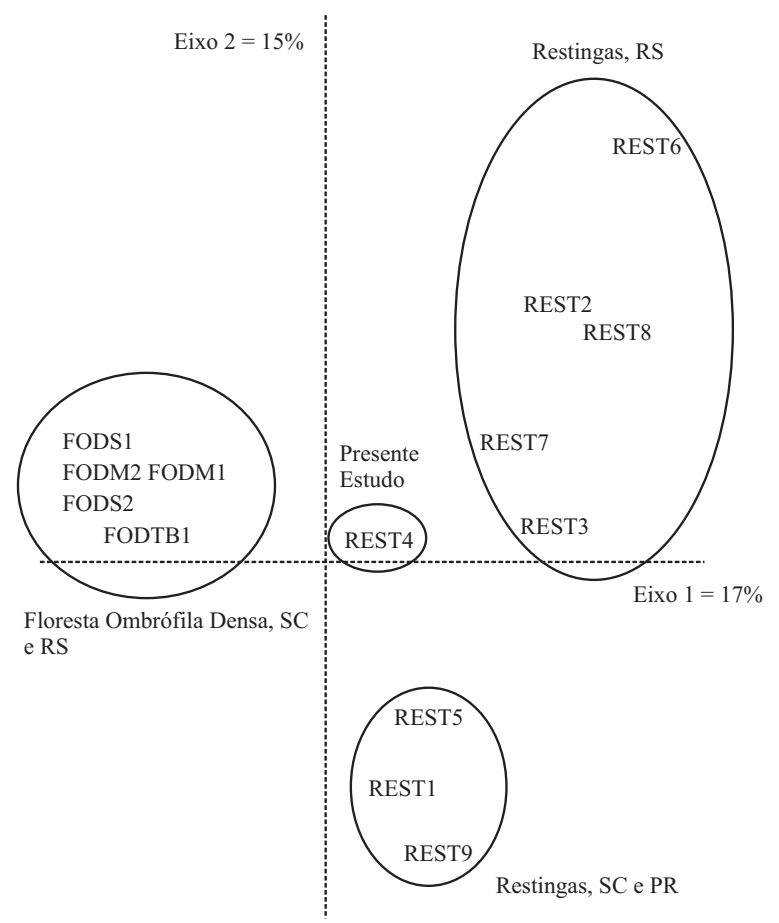

Figura 3 - Análise de correspondência de formações de Floresta Atlântica no sul do Brasil relacionadas na metodologia, onde: FODM Floresta ombrófila densa Montana, FODS Floresta ombrófila densa submontana, FODTB Floresta ombrófila densa das terras baixas, REST Restinga.

Figure 3-Atlantic Forest formations correspondence analyses in the South of Brazil listed in the methodology, where: FODM - Mountain Dense Ombrophilous Forest; FODS - Submountain Dense Ombrophilous Forest; FODTB - Lowlands Dense Ombrophilous Forest, REST Restinga. 
do Sul, através da "Porta de Torres", constituindo-se como o canal de comunicação da Floresta Atlântica entre o Sul de Santa Catarina e o Nordeste do Rio Grande do Sul. Grande parte das espécies do contingente atlântico ocupa o estrato do sub-bosque da floresta.

Os altos valores de densidade obtidos por espécies de ampla distribuição (i.e. S. bonplandii) refletem a elevada plasticidade ecológica dessas espécies e a sua capacidade de adaptação a ambientes estressantes. A colonização de um novo ambiente ocorre normalmente a partir de taxas reprodutivas positivas e de condições ambientais semelhantes, propiciando a capacidade de competição interespecífica que leva à permanência e desenvolvimento da nova espécie na área (PEARMAN et al., 2008). Taxas reprodutivas positivas estão, muitas vezes, relacionadas à interação com agentes polinizadores e dispersores. A dispersão é importante interação entre a fauna e a flora, constituindo um dos mecanismos essenciais para a dinâmica da floresta e, consequentemente, influenciando na regeneração natural das populações (ZAMBONIM, 2001; TABARELLI; PERES, 2002).

A zoocoria, neste estudo, representou $91 \%$ do total de espécies amostradas. Esse padrão também foi identificado por Scherer et al. (2005), em componente arbóreo de restinga arenosa no Parque Estadual de Itapuã, RS. Esses estudos ratificaram a importância dos agentes bióticos no fluxo gênico em restingas arenosas, assemelhando-se ao resultado de vários autores,como o mais relevante modo de dispersão das espécies lenhosas na Floresta Atlântica.

O padrão de distribuição geográfico dos estudos, obtidos por análise de correspondência, remete a proximidade geográfica como primeira causa de maior similaridade entre áreas (padrão geográfico), corroborado, muitas vezes, por condições ambientais similares (clima, temperatura, precipitação, solo etc.), como visto nas formações de Floresta Ombrófila Densa Atlântica. Entretanto, áreas distantes geograficamente, mas com condições ambientais similares, podem também apresentar similaridade em sua composição (GUARINO; WALTER, 2005). Esses padrões podem influenciar na classificação da vegetação, ora baseada em aspectos florísticos (espécies dominantes e frequentes), ora em aspectos ambientais (LIMA et al., 2009).

Analisando os resultados da regeneração natural das espécies arbustivo-arbóreas, observou-se que as 10 primeiras espécies acumularam 54\% da regeneração natural total. Os resultados obtidos pelo RNT na comunidade estudada variaram de $13,5 \%$ a $0,2 \%$. Scherer et al. (2005) obtiveram, em floresta de restinga arenosa, índices de regeneração natural total com amplitudes variando de $22,2 \%$ a $0,6 \%$, superiores aos resultados deste estudo. A ocorrência restrita de algumas espécies no componente regenerante pode indicar um fluxo de novas espécies para a comunidade, ou apontar característica de raridade dessas populações na comunidade ou, ainda, apresentar maior dificuldade em se instalar na comunidade em estudo, devido às suas condições ambientais. Euterpe edulis foi amostrada apenas no componente regenerante, evidenciandose o papel da "Porta de Torres" como importante rota migratória de espécies do contingente atlântico no Rio Grande do Sul. A referida espécie é considerada como representante típico desse contingente florístico, avançando até a porção norte da mata da encosta da Serra do Sudeste e, ao longo da encosta meridional do Planalto Sul-brasileiro, até a região central do Estado (JARENKOW; WAECHTER, 2001).

Os menores índices de regeneração estimados da área em estudo podem ser atribuídos a vários fatores, entre eles o tipo e propriedades do solo, no trecho analisado, pois em Itapeva, Município de Torres, predomina o solo arenoso, com maior deposição de serapilheira nas baixadas entre dunas. Um importante aspecto relacionado à estratégia de regeneração está relacionado à densidade de adultos na comunidade, assim como a estratégia de crescimento. Árvores do dossel necessitam de muito mais recursos para reprodução do que espécies do sub-bosque que normalmente reproduzem na sombra e possuem estrutura morfológica mais simples (COMITA et al., 2007), como evidenciado em S. bonplandii.

Tratando-se de formações pioneiras como as restingas, grande parte das espécies encontradas tem sua ocorrência associada à capacidade de tolerar o estresse causado pelo ambiente, refletindo grande plasticidade ecológica por parte das espécies, não se enquadrando, muitas vezes, em grupos ecológicos típicos aplicados às florestas tropicais.

Relacionando aspectos da regeneração e grupos ecológicos, pode-se estabelecer a tolerância à sombra como importante estratégia na manutenção da alta diversidade e coexistência de espécies, pois estas são capazes de se estabelecer e permanecer por longo 
período no sub-bosque. Na outra extremidade do espectro, plantas dependentes de luz necessitam de "eventos de chance" (BROKAW; BUSING, 2000) como a abertura do dossel para sobrevivência, levando a um menor número de indivíduos no banco de plântulas, em comparação com espécies tolerantes à sombra. Esse padrão conduz à expectativa de maior coexistência de espécies no subbosque em detrimento do dossel. A distinção de estratégias de coexistência a respeito dos estádios do ciclo de vida pode revelar mecanismos que operam dentro e entre os ambientes, fornecendo bases para elucidar a complexa relação nas comunidades vegetais, ao longo de gradientes ambientais (MARTINS, 2010).

Características ecológicas estão cada vez mais presentes nas hipóteses de coexistência, evidenciadas por mecanismos importantes como as permutas obrigatórias (trade off), as formas de vida e as fases ontogenéticas do ciclo de vida, configurando em quatro fatores ambientais e históricos (1) a heterogeneidade horizontal; (2) a heterogeneidade vertical; (3) o regime de distúrbio; e (4) as interações bióticas. Tais fatores contribuem para a coexistência de espécies entre as fases de vida das plantas (NAKASHIZUKA, 2001).

\section{CONCLUSÃO}

Por meio da análise florístico-estrutural e fitogeográfica realizada na restinga e em florestas ombrófilas da Região Sul do Brasil, pode-se afirmar que a restinga em questão possui riqueza específica elevada quando comparada com outros levantamentos regionais, o que se deve, provavelmente, à predominância dos elementos florísticos provindos do contingente Leste e de espécies de ampla distribuição. Pelo elevado número de espécies presentes, estrutura comunitária e regeneração natural constatados, bem como pela contribuição da Floresta Ombrófila Densa Atlântica, obtiveram-se resultados diferentes de outros levantamentos realizados em vegetação de Restinga no Sul do Brasil, evidenciando a importância da "Porta de Torres" como corredor da vegetação para o Rio Grande do Sul.

Nessa direção, este estudo agregou novas informações ao conhecimento sobre a estrutura comunitária da Restinga do Rio Grande do Sul.

Os resultados aqui apresentados contribuirão para posteriores estudos de dinâmica no PEVA e para programas de manejo, preservação e recuperação ambiental.

\section{AGRADECIMENTOS}

A Capes, pelo suporte financeiro representado pela concessão de Bolsa de Mestrado ao primeiro autor.

\section{REFERÊNCIAS}

ARAúJO, D. S. D.; LACERDA, L. D. A natureza das restingas. Ciência Hoje, v.6, n.33, p.42-48, 1987.

ASSUMPÇÃO, J.; NASCIMENTO, M. T. Estrutura e composição florística de quatro formações vegetais de restinga no complexo lagunar Grussaí/Iquipari, São João da Barra, RJ, Brasil. Acta Botanica Brasilica, v. 14, n.3, p.301-315, 2000.

BROKAW, N.; BUSING, R. T. Niche versus chance and tree diversity in forest gaps. Trends in Ecology \& Evolution, v.15, p.183-187, 2000.

CITADINI-ZANETTE, V. Florística, fitossociologia e aspectos da dinâmica de um remanescente de Mata Atlântica na microbacia do Rio Novo, Orleans, SC. 1995. 236f. Tese (Doutorado em Ecologia) - Universidade Federal de São Carlos, São Carlos, 1995.

CITADINI-ZANETTE, V.; SANTOS, R.; SOBRAL, M. Levantamento florístico da vegetação arbustiva-arbórea em área ecotonal entre restinga e floresta ombrófila densa de terras baixas (Praia de Palmas, Governador Celso Ramos, Santa Catarina, Brasil). Revista Tecnologia e Ambiente, v.7, n.1, p.105-120, 2001.

CITADINI-ZANETTE, V. et al. Myrtaceae do sul de Santa Catarina: subsídio para recuperação de ecossistemas degradados. Revista de

Tecnologia e Ambiente, v.9, n.2, p.61-75, 2003.

COMITA, L. S. et al. Patterns of woody plant species abundance and diversity in the seedling layer of a tropical forest. Journal of

Vegetation Science, v.18, p.163-174, 2007.

DILLENBURG, L. R.; WAECHTER, J. L.; PORTO, M. L. Species composition and structure of a sandy coastal plain forest in northern Rio Grande do Sul, Brazil. In: SEELIGER, U. (Org.). Coastal plant communities of Latin America. San Diego: Academic Press, 1992. p.349-366.

Revista Árvore, Viçosa-MG, v.36, n.6, p.1047-1059, 2012 
DORNELES, L. P. P.; WAECHTER, J. L. Estrutura do componente arbóreo da floresta arenosa de restinga do Parque Nacional da Lagoa do Peixe, Rio Grande do Sul. Hoehnea, v.31, n.1, p.61-71, 2004.

FALKENBERG, D. B. Aspecto da flora e da vegetação secundária da Restinga de Santa Catarina, sul do Brasil. Insula, n. 28, p.1-30, 1999.

FERRETTI, A. R. et al. Classificação das espécies arbóreas em grupos ecológicos para revegetação com nativas no Estado de São Paulo. Florestar Estatístico, v.3, n.7, p.73-77, 1995.

FELFILI, J. M. et al. Procedimentos e métodos de amostragem de vegetação. In: FELFILI, J. M. et al. (Org.). Fitossociologia no Brasil: métodos e estudos de casos. Viçosa, MG: Universidade Federal de Viçosa, 2011. p.86-121.

FINOL, U. H. Nuevos parámetros a considerarse en el análisis estructural de las selvas vírgenes tropicales. Revista Forestal Venezolana, v.14, n.21, p.29-42, 1971.

FORZZA, R. C. et al. (Org.). Catálogo de plantas e fungos do Brasil. Rio de Janeiro: Instituto de Pesquisas Jardim Botânico do Rio de Janeiro, 2010. 1699 p.

FREIRE, M. S. B. Levantamento florístico do Parque Estadual das Dunas de Natal. Acta Botanica Brasilica, v. 4, n. 2, p. 41-59, 1990.

GIEHL, E. L. H.; JARENKOW, J. A. Gradiente estrutural no componente arbóreo e relação com inundações em uma floresta ribeirinha, rio Uruguai, sul do Brasil. Acta Botanica Brasilica, v.22, n.3, p.741-753, 2008.

GUARINO, E. S. G.; WALTER, B. M. T. Fitossociologia de dois trechos inundáveis de matas de galeria no Distrito Federal, Brasil. Acta Botanica Brasilica, v. 19, n.3, p.431-442, 2005.

HENSTSCHEL, R. L. Gradiente vegetacional, variavéis ambientais e restauração na restinga da Praia do Ouvidor, Garopaba, Santa Catarina. 2008. $76 f$. Dissertação (Mestrado em Botânica) - Universidade Federal do Rio Grande do Sul, Porto Alegre, 2008.

\section{INSTITUTO NACIONAL DE METEOROLOGIA, MINISTÉRIO DA AGRICULTURA, PECUÁRIA E}

ABASTECIMENTO - INMET. Disponível em: <http://www.inmet.gov.br>. Acesso em: 28 out. 2009.

JARENKOW, J. A. Estudo fitossociológico comparativo entre duas áreas com mata de encosta no Rio Grande do Sul. 1994. 122f. Tese (Doutorado em Ecologia e Recursos Naturais) - Universidade Federal de São Carlos, São Carlos, 1994.

JARENKOW, J. A.; WAECHTER, J. L. Composição, estrutura e relações florísticas do componente arbóreo de uma floresta estacional no Rio Grande do Sul, Brasil. Revista Brasileira de Botânica, v.24, n.3, p.263-272, 2001.

KENT, M.; COKER, P. Vegetation description and analysis. Nova York: Wiley, 1992. 363 p.

LIMA, J. R. et al. Composição florística da Floresta Estacional Decídua Montana de Serra das Almas, CE, Brasil. Acta Botanica Brasilica, v.23, n.3, p.756-763, 2009.

LINDEMAN, C. A. M. Vegetação no Rio Grande do Sul. Porto Alegre: Universal, 1906. 365 p.

MAGNAGO, L. F. S.; MARTINS, S. V.; PEREIRA, O. J. Heterogeneidade florística das fitocenoses de restingas nos estados do Rio de Janeiro e Espírito Santo, Brasil. Revista Árvore, v.35, n.2, p.245-254, 2011.

MAGNAGO, L. F. S. et al. Gradiente fitofisionômico-edáfico em formações florestais de Restinga no sudeste do Brasil. Acta Botanica Brasilica, v.24, n.3, p.734-746, 2010.

MARTINS, R. Florística, estrutura fitossociológica e interações interespecíficas de um remanescente de Floresta Ombrófila Densa como subsídio para recuperação de áreas degradadas pela mineração de carvão, Siderópolis, SC. 2005. 93f. Dissertação (Biologia Vegetal) - Universidade Federal de Santa Catarina, Florianópolis, 2005.

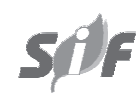

Revista Árvore, Viçosa-MG, v.36, n.6, p.1047-1059, 2012 
MARTINS, R. Composição e estrutura vegetacional em diferentes formações na floresta atlântica, sul de Santa Catarina, Brasil. 2010. 148f. Tese (Doutorado em Botânica)- Universidade Federal do Rio Grande do Sul, Porto Alegre, 2010.

MORAES, D.; MONDIN, C. A. Florística e fitossociologia do estrato arbóreo em mata arenosa no Balneário Quintão, Palmares do Sul/ RS. Pesquisas, Série Botânica, n.51, p.87-100, 2001.

MORETTO, F.; MONDIN C. A. Levantamento quali-quantitativo do componente lenhoso do sub-bosque de uma mata arenosa de restinga no Balneário Quintão, Palmares do Sul/RS, Porto Alegre. Pesquisas, Série Botânica, n.52, p.111-123, 2002.

MUELLER-DOMBOIS, D.; ELLENBERG, H. Aims and methods of vegetation ecology. New York: Wiley, 1974. 574 p.

MURRAY-SMITH, C. et al. Plant diversity hotspots in the atlantic coastal forests of Brazil. Conservation Biology, v.23, n.1, p.151-163, 2008.

NAKASHIZUKA, T. Species coexistence in temperate, mixed deciduous forests. Trends in Ecology \& Evolution, v.16, n.4, p.205-210, 2001.

NEGRELLE, R. R. B. Composição florística e estrutura vertical de um trecho de Floresta Ombrófila Densa de Planície Quaternária. Hoehnea, v.33, n.3, p.261-289, 2006.

PEARMAN, P. B. et al. Niche dynamics in space and time. Trends in Ecology \& Evolution, v.23, n.3, p.149-158, 2008.

PORTO ALEGRE. Secretaria Estadual do Meio Ambiente do Rio Grande do Sul - SEMA. Plano de manejo do Parque Estadual de Itapeva. Porto Alegre: 2006. 259 p.

RAMBO, B. A porta de torres. Anais Botânicos do Herbário Barbosa Rodrigues, n.2, p.9-20, 1950.
RAMBO, B. História da flora do litoral riograndense. Sellowia, n.6, p.113-172, 1954.

RIO GRANDE DO SUL. Decreto n. ?42099 de 31 de dezembro de 2002. Lista de espécies da flora nativa ameaçadas de extinção no Rio Grande do Sul. Diário Oficial do Estado do Rio Grande do Sul, p.1-6, 2003.

ROCHA, C. F. D. et al. Endemic and threatened tetrapods in the restingas of the biodiversity corridors of Serra do Mar and of the central da Mata Atlântica in Eastern. Brazilian Journal of Biology, v.65, n.1, p.159-168, 2005.

ROSSONI, M. G.; BAPTISTA, L. R. M.

Composição florística da mata de restinga, Balneário Rondinha Velha, Arroio do Sal, RS, Brasil. Pesquisas, Série Botânica, n.45, p.115-131, 1995.

SACRAMENTO, A. C.; ZICKEL, C. S.; ALMEIDA JUNIOR, E. B. Aspectos florísticos de restinga no litoral de Pernambuco. Revista Árvore, v.31, n.6, p.1121-1130, 2007.

SCHERER, A.; MARASCHIN-SILVA, F.; BAPTISTA, L. R. M. Regeneração arbórea num capão de restinga no Rio Grande do Sul, Brasil. Iheringia, Série Botânica, v.62, n.1-2, p.89-98, 2007.

SCHERER, A.; SILVA, F. M.; BAPTISTA, L. R. M. Florística e estrutura do componente arbóreo de matas de restinga arenosa no Parque de Itapuã, RS, Brasil. Acta Botanica Brasilica, v.19, n.4, p.717-726, 2005.

SIlva, S. M. Composição florística e fitossociologia de um trecho de floresta de restinga na Ilha do Mel, Paranaguá, PR. 1990. 146f. Dissertação (Mestrado em Biologia Vegetal)- Universidade Estadual de Campinas, São Paulo, 1990.

SILVA, S. M.; BRITEZ, R. M. A vegetação da Planície Costeira. In: MARQUES, M. C. M.; BRITEZ, R. M. (Org.). História natural e conservação da Ilha do Mel. Curitiba: Universidade Federal do Paraná, 2005. p.49-84. 
TABARELLI, M.; PERES, C. A. Abiotic and vertebrate seed dispersal in the Brazilian Atlantic Forest: implications for forest regeneration. Biological Conservation, v.106, p.165-176, 2002.

van der PIJL, L. Principles of dispersal in higher plants. 2.ed. Berlin: Springer, 1972. 215 p.

VOLPATO, M. M. L. Regeneração natural em uma floresta secundária no domínio de Mata Atlântica: uma análise fitossociológica. 1994. 123f. Dissertação (Mestrado)- Universidade Federal de Viçosa, Viçosa, MG, 1994.
WAECHTER, J. L. Aspectos ecológicos da vegetação de restinga no Rio Grande do Sul, Brasil. Comunicações do Museu de Ciências da PUCRS, Série Botânica, n.33, p.49-68, 1985.

ZAMBONIM, R. M. Banco de dados como subsídio para conservação e restauração nas tipologias vegetacionais do Parque Estadual da Serra do Tabuleiro. 2001. 118f. Dissertação (Mestrado em Recursos Genéticos Vegetais) Universidade Federal de Santa Catarina, Florianópolis, 2001. 
